\title{
Three Results on Cycle-Wheel Ramsey Numbers
}

\author{
Yanbo Zhang • Hajo Broersma • Yaojun Chen
}

Received: 3 June 2014 / Revised: 25 November 2014 / Published online: 21 January 2015

C The Author(s) 2015. This article is published with open access at Springerlink.com

\begin{abstract}
Given two graphs $G_{1}$ and $G_{2}$, the Ramsey number $R\left(G_{1}, G_{2}\right)$ is the smallest integer $N$ such that, for any graph $G$ of order $N$, either $G_{1}$ is a subgraph of $G$, or $G_{2}$ is a subgraph of the complement of $G$. We consider the case that $G_{1}$ is a cycle and $G_{2}$ is a (generalized) wheel. We expand the knowledge on exact values of Ramsey numbers in three directions: large cycles versus wheels of odd order; large wheels versus cycles of even order; and large cycles versus generalized odd wheels.
\end{abstract}

Keywords Ramsey number $\cdot$ Cycle $\cdot$ Wheel

\section{Introduction}

In this paper we deal with finite simple graphs only. For any undefined terminology and notation we refer the reader to the textbook of Bondy and Murty [3].

Let $G=(V, E)$ be a graph. We sometimes use $|G|$ instead of $|V|$ to denote the order of $G$, i.e., the number of vertices of $G$. For a nonempty proper subset $S \subseteq V(G)$, we let $G[S]$ and $G-S$ denote the subgraphs induced by $S$ and $V(G) \backslash S$, respectively. Let $N_{S}(v)$ be the set of neighbors of a vertex $v$ that are contained in $S, N_{S}[v]=N_{S}(v) \cup\{v\}$ and $d_{S}(v)=\left|N_{S}(v)\right|$. If $S=V(G)$, we sometimes simply write $N(v)=N_{G}(v)$, $N[v]=N(v) \cup\{v\}$ and $d(v)=d_{G}(v)$. For two vertex-disjoint graphs $G_{1}$ and $G_{2}$, $G_{1} \cup G_{2}$ denotes the disjoint union, and the join $G_{1}+G_{2}$ is the graph obtained from

\footnotetext{
Y. Zhang · Y. Chen

Department of Mathematics, Nanjing University, Nanjing 210093, People's Republic of China

Y. Zhang $\cdot$ H. Broersma $(\varangle)$

Faculty of Electrical Engineering, Mathematics and Computer Science,

University of Twente, P.O. Box 217, 7500 AE Enschede, The Netherlands

e-mail: h.j.broersma@utwente.nl
} 
$G_{1} \cup G_{2}$ by joining every vertex of $G_{1}$ to every vertex of $G_{2}$ by an edge. A cycle and a path of order $m$ are denoted by $C_{m}$ and $P_{m}$, respectively. We sometimes use $C_{m}$ instead of $V\left(C_{m}\right)$ for simplicity. By $x P_{m} y$ we mean a path from $x$ to $y$ with order $m$. An $(X, Y)$ path is a path that starts at a vertex of $X$, and terminates at a vertex of $Y$. We use $K_{n}$ to denote a complete graph of order $n$, and $K_{m, n}$ for a complete bipartite graph with bipartition classes of cardinality $m$ and $n$. A wheel $W_{n}=K_{1}+C_{n}$ is a graph of order $n+1$ (in the literature, sometimes $W_{n}$ is used to denote a wheel of order $n)$, and a generalized wheel $W_{m, n}=K_{m}+C_{n}$, so that $W_{1, n}=W_{n}$. As in [3], $\delta(G)$ is the minimum degree, $\Delta(G)$ the maximum degree, $\alpha(G)$ the independence number and $\kappa(G)$ the (vertex) connectivity of $G$. We use $m G$ to denote $m$ vertex-disjoint copies of $G$. The length of a longest and shortest cycle of $G$ is denoted by $c(G)$ and $g(G)$, respectively. A graph $G$ is weakly pancyclic if it contains cycles of every length between $g(G)$ and $c(G)$. We say that $G$ is Hamilton-connected if every two distinct vertices of $G$ are connected by a Hamilton path.

Given two graphs $G_{1}$ and $G_{2}$, the Ramsey number $R\left(G_{1}, G_{2}\right)$ is the smallest integer $N$ such that, for any graph $G$ of order $N$, either $G$ contains $G_{1}$ as a subgraph or $\bar{G}$ contains $G_{2}$ as a subgraph, where $\bar{G}$ is the complement of $G$. It is easy to check that $R\left(G_{1}, G_{2}\right)=R\left(G_{2}, G_{1}\right)$. For specific graphs or graph families, the greatest challenge is to determine the exact values of the Ramsey numbers. This challenging open problem was solved for cycles by Rosta [17], and independently by Faudree and Schelp [13], as shown by the following result. A simpler proof for this result was later provided by Károlyi and Rosta [15].

Theorem 1 (Rosta [17], Faudree and Schelp [13]).

$$
R\left(C_{m}, C_{n}\right)= \begin{cases}2 n-1 & \text { for } 3 \leq m \leq n, m \text { odd, } n \neq 3, \\ n-1+m / 2 & \text { for } 4 \leq m \leq n, m \text { and } n \text { even, } n \neq 4 \\ \max \{n-1+m / 2,2 m-1\} & \text { for } 4 \leq m<n, m \text { even and } n \text { odd }\end{cases}
$$

Wheels have also enjoyed quite a lot of attention in the context of Ramsey numbers. In the earliest contribution involving cycle-wheel Ramsey numbers, dating back to 1983, Burr and Erdős [6] determined the Ramsey numbers of a triangle versus wheels of arbitrarily large order.

Theorem 2 (Burr and Erdős [6]). $R\left(W_{n}, C_{3}\right)=2 n+1$ for $n \geq 5$.

From then on, many papers have been published on cycle-wheel Ramsey numbers. By comparing the order of the two graphs and the parity of the smaller one, we can distinguish four cases: large cycles versus wheels of even (odd) order and large wheels versus cycles of even (odd) order. We recall here that a wheel $W_{n}=K_{1}+C_{n}$ is a graph of order $n+1$, so even (odd) wheels correspond to odd (even) $n$.

For large cycles versus even wheels, Surahmat et al. [22] determined that $R\left(C_{m}, W_{n}\right)=3 m-2$ for odd $n \geq 5$ and $m>(5 n-9) / 2$. This result was improved by Shi [18] who showed that $R\left(C_{m}, W_{n}\right)=3 m-2$ for odd $n$ and $m \geq 3 n / 2+1$. Then Zhang et al. [28] refined that result to $R\left(C_{m}, W_{n}\right)=3 m-2$ for odd $n, m \geq n$ and $m \geq 20$. Finally, Chen et al. gave a simpler proof that completely solves this case. 
Theorem 3 (Chen et al. [8]). $R\left(C_{m}, W_{n}\right)=3 m-2$ for odd $n, m \geq n \geq 3$ and $(m, n) \neq(3,3)$.

For large cycles versus odd wheels, Surahmat et al. [21] proved that $R\left(C_{m}, W_{n}\right)=$ $2 m-1$ for even $n$ and $m \geq 5 n / 2-1$. Chen et al. [7] improved this result by reducing the lower bound on $m$ from $m \geq 5 n / 2-1$ to $m \geq 3 n / 2+1$. To completely solve this case, Surahmat et al. [21] proposed the following conjecture.

Conjecture 1 (Surahmat et al. [21]). $R\left(C_{m}, W_{n}\right)=2 m-1$ for even $n, m \geq n$ and $(m, n) \neq(4,4)$.

We will confirm the above conjecture for large even $m$ in this paper by proving the following result. We postpone the proof to the final section.

Theorem $4 R\left(C_{m}, W_{n}\right)=2 m-1$ for even $n$ and $m \geq n+502$.

For large wheels versus cycles of odd order, besides Theorem 2 on triangles versus wheels of arbitrarily large order, Zhou [31] showed that $R\left(W_{n}, C_{m}\right)=2 n+1$ for $m$ odd and $n \geq 5 m-7$. Even though it has been cited many times, the correctness of the proof in this Chinese language paper is questionable. Recently, it was established by Sun and Chen [19] that $R\left(W_{n}, C_{5}\right)=2 n+1$ for $n \geq 6$. And Zhang et al. [30] proved the following more general result.

Theorem 5 (Zhang et al. [30]). $R\left(W_{n}, C_{m}\right)=2 n+1$ for $m$ odd, $n \geq 3(m-1) / 2$ and $(m, n) \neq(3,3),(3,4) ; R\left(W_{n}, C_{m}\right)=3 m-2$ for $m, n$ odd and $m<n<3(m-1) / 2$.

For large wheels versus small cycles of even order, even $R\left(C_{4}, W_{n}\right)$ is quite challenging. Tse [25] determined the value of $R\left(C_{4}, W_{n}\right)$ for $3 \leq n \leq 12$. Surahmat et al. [23] established an upper bound, which is $R\left(C_{4}, W_{n}\right) \leq n+\lceil n / 3\rceil+1$ for $n \geq 6$. Recently, Dybizbański and Dzido [10] refined the upper bound and determined some exact values of $R\left(C_{4}, W_{n}\right)$.

Theorem 6 (Dybizbański and Dzido [10]). $R\left(C_{4}, W_{n}\right) \leq n+\lfloor\sqrt{n-1}\rfloor+2$ for all $n \geq 10$, and if $q \geq 4$ is a prime power, then $R\left(C_{4}, W_{q^{2}}\right)=q^{2}+q+1$.

In the same paper, they proved that $R\left(C_{4}, W_{n}\right)=n+5$ for $13 \leq n \leq 16$. Wu et al. [26] went a step further and obtained nine new values of $R\left(C_{4}, W_{n}\right)$ for $17 \leq n \leq 20$, $34 \leq n \leq 36$ and $n=26,43$.

By comparing the Ramsey numbers of $R\left(C_{4}, K_{1, n}\right)$ and $R\left(C_{4}, W_{n}\right)$, we answered a natural question affirmatively by proving the following theorem in a very recent paper [29].

Theorem 7 (Zhang et al. [29]). $R\left(C_{4}, K_{1, n}\right)=R\left(C_{4}, W_{n}\right)$ for $n \geq 6$.

By Theorem 7, we see that the values for $R\left(C_{4}, K_{1, n}\right)$ and $R\left(C_{4}, W_{n}\right)$ are exactly the same for $n \geq 6$. Thus we can use known results on $R\left(C_{4}, K_{1, n}\right)$ to obtain new values for $R\left(C_{4}, W_{n}\right)$ immediately. This yielded the following theorem.

Theorem 8 (Zhang et al. [29]). If $q \geq 3$, then $R\left(C_{4}, W_{q^{2}+1}\right) \leq q^{2}+q+2$. If $q \geq 3$ is a prime power, then $R\left(C_{4}, W_{q^{2}+1}\right)=q^{2}+q+2$. 
A weaker version of Theorem 8 was established by Wu et al. [27] independently. They also obtained some more values.

Theorem 9 (Wu et al. [27]). If $q \geq 5$ is a prime power, then $R\left(C_{4}, W_{q^{2}-2}\right)=q^{2}+q-$ 1. If $p \geq 3, q=2^{p}, 0 \leq k \leq q$ and $k \neq 1, q-1$, then $R\left(C_{4}, W_{q^{2}-k-1}\right)=q^{2}+q-k$.

As far as we know, practically nothing is known about $R\left(W_{n}, C_{m}\right)$ when $m$ is even and greater than 4 . We fill some of this gap by proving the following result in the final section.

Theorem $10 R\left(W_{n}, C_{m}\right)=3 m-2$ for $n$ odd, $m$ even and $m<n<3 m / 2$.

For large cycles versus generalized even wheels, Surahmat et al. [24] showed that $R\left(C_{m}, W_{2, n}\right)=3 m-2$ for even $n \geq 4$ and $m \geq 9 n / 2+1$. Shi [18] improved this result by reducing the lower bound on $m$ from $m \geq 9 n / 2+1$ to $m \geq \max \{3 n / 2+1,71\}$. For large cycles versus generalized odd wheels, in the final section we prove the following result that has the same flavor.

Theorem $11 R\left(C_{m}, W_{2, n}\right)=4 m-3$ for $n$ odd, $m \geq 9 n / 8+1$.

\section{Preliminary Lemmas}

For our proofs of Theorems 4, 10 and 11 in the next section, we need the following useful lemmas. Except for one (Lemma 15 below), all results are from literature and presented without proofs.

Lemma 1 (Bollobás et al. [1]). $R\left(C_{n}, K_{5}\right)=4 n-3$ for $n \geq 5$.

Lemma 2 (Bondy [2]). Let $G$ be a graph of order $n$. If $\delta(G) \geq n / 2$, then either $G$ is pancyclic or $n$ is even and $G=K_{n / 2, n / 2}$.

Lemma 3 (Brandt [4]). Every nonbipartite graph $G=(V, E)$ of order $n$ with $|E|>$ $(n-1)^{2} / 4+1$ is weakly pancyclic with $g(G)=3$.

Lemma 4 (Brandt et al. [5]). Every nonbipartite graph $G$ of order $n$ with $\delta(G) \geq$ $(n+2) / 3$ is weakly pancyclic with $g(G)=3$ or 4 .

Lemma 5 (Brandt et al. [5]). Let $G$ be a 2-connected nonbipartite graph of order $n$ with minimum degree $\delta(G) \geq n / 4+250$. Then $G$ is weakly pancyclic unless $G$ has odd girth 7, in which case it has cycles of every length from 4 up to its circumference except a 5-cycle.

Lemma 6 (Dirac [9]). Let $G$ be a graph with $\delta(G) \geq 2$. Then $c(G) \geq \delta(G)+1$. If $G$ is 2-connected, then $c(G) \geq \min \{2 \delta(G),|V(G)|\}$.

Lemma 7 (Dirac [9]). Let $G$ be a graph of order $n$. If $\delta(G) \geq n / 2+1$, then $G$ is Hamilton-connected.

Lemma 8 (Erdős and Gallai [11]). Let $G=(V, E)$ be a graph of order $n$ and $3 \leq$ $c \leq n$. If $|E| \geq((c-1)(n-1)+1) / 2$, then $c(G) \geq c$. 
Lemma 9 (Faudree et al. [12]). Let $G$ be a graph of order $n \geq 6$. Then $\max \{c(G), c(\bar{G})\} \geq\lceil 2 n / 3\rceil$.

Lemma 10 (Jackson [14]). Let $G=(X, Y)$ be a bipartite graph with bipartition classes $X$ and $Y$ such that $d(x) \geq t$ for all $x \in X$, where $|X| \geq 2$ and $2 \leq t \leq|Y| \leq$ $2 t-2$. Then $G$ contains all cycles on $2 m$ vertices for $2 \leq m \leq \min \{|X|, t\}$.

Lemma 11 (Károlyi and Rosta [15]). Suppose that $G$ has a cycle $C=x_{1} x_{2} \ldots x_{2 \ell} x_{1}$, but neither $G$ nor $\bar{G}$ has a $C_{2 \ell-1}$. Then $\bar{G}\left[\left\{x_{1}, x_{3}, \ldots, x_{2 \ell-1}\right\}\right]=\bar{G}\left[\left\{x_{2}, x_{4}, \ldots, x_{2 \ell}\right\}\right]$ $=K_{\ell}$.

The following lemma can be found as Proposition 9.4 in [3].

Lemma 12 Let $G$ be a k-connected graph, and let $X$ and $Y$ be subsets of $V(G)$ of cardinality at least $k$. Then there exists a family of $k$ pairwise disjoint $(X, Y)$ paths in $G$.

Lemma 13 (Zhang et al. [30]) Let $C$ be a longest cycle in a graph $G$ and $v_{1}, v_{2} \in$ $V(G) \backslash V(C)$ with $t=\left|N_{C}\left(v_{1}\right) \cup N_{C}\left(v_{2}\right)\right|$. Then $t \leq\lfloor|C| / 2\rfloor+1$ and if $v_{1} v_{2} \in E(G)$, then $t \leq\lfloor|C| / 2\rfloor$.

Lemma 14 (Zhang et al. [30]) For a graph $G$, let $(X, Y)$ be a partition of $V(G)$. Suppose that for some odd $n \geq 5,|Y| \geq(n+1) / 2$ and any two vertices of $Y$ have at least $(n-1) / 2$ common nonadjacent vertices in $X$. If $\bar{G}$ contains no $C_{n}$, then $G[Y]$ is a complete graph.

Lemma 15 Let $C=x_{1} x_{2} \ldots x_{r} x_{1}$ be a longest cycle in a graph $G$ with $r \geq n$, and let $Y=\left\{y_{1}, y_{2}, \ldots, y_{d-r}\right\}=V(G) \backslash V(C)$ with $d-r \geq(n+1) / 2$. Suppose that $\bar{G}$ contains no $C_{n}$ and $G[Y]$ is a complete graph. Then $\bar{G}$ is bipartite.

Proof If $E(V(C), Y)=\emptyset$, then $G[V(C)]$ is a complete graph by Lemma 14. Hence, in this case it is easy to deduce that $\bar{G}$ is bipartite. Now let $E(V(C), Y) \neq \varnothing$, and let $x_{1} y_{1} \in E(G)$. Then, since $C$ is a longest cycle in $G$ and $G[Y]$ a complete graph, it follows that $E\left(X_{1}, Y \backslash\left\{y_{1}\right\}\right)=\emptyset$, where $X_{1}=\left\{x_{2}, x_{3}, \ldots, x_{d-r+1}\right\} \cup$ $\left\{x_{r}, x_{r-1}, \ldots, x_{2 r-d+1}\right\}$. Because $d-r-1 \geq(n-1) / 2$, using Lemma 14 again, we obtain that $G\left[X_{1}\right]$ is a complete graph. Let $H \subseteq G[V(C)]$ be a maximal complete graph containing $x_{2}$. We claim that $V(C) \backslash\left\{x_{1}\right\} \subseteq V(H)$. If not, there is an $x_{i} \in V(C) \backslash V(H)$ such that $x_{i}$ is adjacent to some vertex of $H$ on the cycle and nonadjacent to some vertex of $H$. Then $E\left(\left\{x_{i}\right\}, Y \backslash\left\{y_{1}\right\}\right)=\emptyset$; otherwise there clearly is a longer cycle than $C$. Furthermore, $\bar{G}\left[V(H) \cup\left(Y \backslash\left\{y_{1}\right\}\right) \cup\left\{x_{i}\right\}\right]$ contains a $C_{n}$, a contradiction. For the same reason, we have $V(C) \backslash\left\{x_{1}\right\} \subseteq N\left(x_{1}\right)$ or $Y \backslash\left\{y_{1}\right\} \subseteq N\left(x_{1}\right)$. Therefore, $\bar{G}$ is bipartite.

Lemma 16 (Surahmat et al. [20]). $R\left(C_{n}, W_{4}\right)=2 n-1$ for $n \geq 5$.

At the end of this section, we list some known small Ramsey numbers that we need.

Lemma 17 ([16]).

(i) $R\left(W_{n}, C_{3}\right)=2 n+3$ for $n=3,4$;

(ii) $R\left(W_{5}, C_{4}\right)=10$. 


\section{Proofs of the Main Results}

\subsection{Proof of Theorem 4}

Let $G$ be a graph of order $2 m-1$ with $n$ even and $m \geq n+502$. Suppose to the contrary that neither $G$ contains $W_{n}$ nor $\bar{G}$ contains $C_{m}$.

Assume that $v \in V(G)$ with $d(v)=d=\Delta(G)$ and $H=G[N(v)]$. We are first going to show that $d \geq(3 m+1) / 2-252$. To the contrary, assume that $d \leq 3 m / 2-252$. Then $\delta(\bar{G}) \geq 2 m-2-(3 m / 2-252)=m / 2+250$. By Theorem 2 and Lemma 17 , we have $g(\bar{G})=3$, and so $\bar{G}$ is nonbipartite. If $\kappa(\bar{G}) \geq 2$, then $\bar{G}$ contains $C_{m}$ by Lemmas 6 and 5 , a contradiction. So, we assume next that $\kappa(\bar{G}) \leq 1$. Then there exists some $u \in V(G)$ such that $G-u$ contains a spanning complete bipartite graph with bipartite sets $V_{1}, V_{2}$ and $\left|V_{1}\right| \geq\left|V_{2}\right|$. Obviously, $\left|V_{1}\right| \geq m-1$ and $\left|V_{2}\right| \geq \delta(\bar{G})$. If $\Delta\left(G\left[V_{1}\right]\right) \geq n / 2$, let $x \in V_{1}$ with $d(x)=\Delta\left(G\left[V_{1}\right]\right)$. Then $x$ together with $n / 2$ neighbors in $V_{1}$ and $n / 2$ neighbors in $V_{2}$ form a $W_{n}$ with $x$ as its hub, a contradiction. This implies that $\delta\left(\bar{G}\left[V_{1}\right]\right) \geq\left|V_{1}\right|-n / 2>\left|V_{1}\right| / 2+1$. If $\left|V_{1}\right| \geq m$, then by Lemma $2, \bar{G}\left[V_{1}\right]$ contains $C_{m}$, a contradiction. Thus, we conclude that $\left|V_{1}\right|=\left|V_{2}\right|=m-1$. Since $\delta(\bar{G})>250$, we may assume that $x_{1}, x_{2} \in N_{\bar{G}}(u) \cap V_{1}$. By Lemma $7, \bar{G}\left[V_{1}\right]$ has a Hamilton $\left(x_{1}, x_{2}\right)$-path which together with $u$ forms $C_{m}$ in $\bar{G}$, our final contradiction. Therefore, henceforth we assume that $d \geq(3 m+1) / 2-252$.

By the assumptions, $H$ has no $C_{n}$. If $m$ is even, then since $m \geq n+502$, we get that $R\left(C_{n}, C_{m}\right) \leq 3 m / 2-252$ by Theorem 1 , a contradiction. Thus, $m$ is odd. Next, we first prove the following claim.

Claim A. $H$ contains no $2 K_{(m+1) / 2}$.

Proof Suppose that $H$ contains $2 K_{(m+1) / 2}$ with $V_{1}, V_{2}$ as their vertex sets.

If there exist two independent edges between $V_{1}$ and $V_{2}$, then $H$ contains a $C_{n}$, a contradiction. If there is at least one edge between $V_{1}$ and $V_{2}$, then $V_{1}$ or $V_{2}$ contains a vertex $w$ such that $E\left(V_{1} \backslash\{w\}, V_{2} \backslash\{w\}\right)=\emptyset$. For any $u \notin V_{1} \cup V_{2} \backslash\{w\}$, we have $V_{1} \backslash\{w\} \subseteq N(u)$ or $V_{2} \backslash\{w\} \subseteq N(u)$, for otherwise $\bar{G}\left[V_{1} \cup V_{2} \cup\{u\}\right]$ contains $C_{m}$. Let $U_{i}=\left\{u \mid V_{i} \backslash\{w\} \subseteq N(u), u \notin V_{1} \cup V_{2} \backslash\{w\}\right\}$ for $i=1,2$. Assume that $\mid\left(V_{1} \backslash\{w\}\right) \cup$ $U_{1}|\geq|\left(V_{2} \backslash\{w\}\right) \cup U_{2} \mid$. Then $\left|\left(V_{1} \backslash\{w\}\right) \cup U_{1}\right| \geq m \geq n+502$. Taking $n+1$ vertices from $\left(V_{1} \backslash\{w\}\right) \cup U_{1}$ such that at least $n / 2+1$ of them are in $V_{1} \backslash\{w\}$, we obtain $W_{n}$ in $G\left[\left(V_{1} \backslash\{w\}\right) \cup U_{1}\right]$, a contradiction. If there is no edge between $V_{1}$ and $V_{2}$, we can derive a contradiction in a similar way.

We next show that $H$ is nonbipartite. To the contrary, suppose $H$ is a bipartite graph with $V(H)=(X, Y)$ and $|X| \geq|Y|$. Since $\bar{G}$ contains no $C_{m}$, we have $|X| \leq m-1$ and $|Y|=d-|X| \geq(m+1) / 2-251>n / 2$. If there exists two independent edges between $X$ and $Y$ in $\bar{G}$, then since both $\bar{G}[X]$ and $\bar{G}[Y]$ are complete graphs, $\bar{H}$ contains $C_{m}$, a contradiction. Thus, there exists some vertex $z \in X \cup Y$ such that $H-z$ with bipartition $(X \backslash\{z\}, Y \backslash\{z\})$ is a complete bipartite graph. But then $H-z$ contains $K_{n / 2, n / 2}$, and so $H$ contains $C_{n}$, a contradiction. Therefore, henceforth we assume that $H$ is nonbipartite.

We now show that $\bar{H}$ is also nonbipartite. To the contrary, suppose $\bar{H}$ is a bipartite graph with $V(\bar{H})=(X, Y)$ and $|X| \geq|Y|$. Since $G[X]$ contains no $C_{n}$, we have $|X| \leq n-1 \leq m-503$ and hence $|Y|=d-|X| \geq(m+1) / 2+251$. Clearly, 
$H$ contains $2 K_{(m+1) / 2}$, contradicting Claim A. Thus, henceforth we assume that $\bar{H}$ is nonbipartite.

If $|E(\bar{H})|>(d-1)^{2} / 4+1$, then by Lemma $3, \bar{H}$ is weakly pancyclic with $g(\bar{H})=3$. By Theorem $1, R\left(C_{n}, C_{m+1}\right)=m+n / 2 \leq(3 m+1) / 2-252$ for $m$ is odd and $m \geq n+502$. Since $H$ contains no $C_{n}, \bar{H}$ contains $C_{m+1}$, which implies that $\bar{H}$ contains $C_{m}$, a contradiction. Thus, we have $|E(H)| \geq d(d-1) / 2-(d-1)^{2} / 4-1>$ $(d-1)^{2} / 4+1$. By Lemma $3, H$ is weakly pancyclic with $g(H)=3$. Since $H$ contains no $C_{n}$, we have $c(H)<n$ and so $H$ has no $C_{m}$. Because $\bar{H}$ contains $C_{m+1}$ and has no $C_{m}, H$ contains $2 K_{(m+1) / 2}$ by Lemma 11 , contradicting Claim A.

Since $2 K_{m-1}$ contains no $C_{m}$ and its complement contains no $W_{n}$, we obtain that $R\left(C_{m}, W_{n}\right) \geq 2 m-1$. By the above arguments, $R\left(C_{m}, W_{n}\right) \leq 2 m-1$ for $n$ even and $m \geq n+502$. This completes the proof of Theorem 4 .

\subsection{Proof of Theorem 10}

By Lemma 17, we may assume that $m \geq 6$. The lower bound $R\left(W_{n}, C_{m}\right) \geq 3 m-2$ follows from the fact that a complete tripartite graph $K_{m-1, m-1, m-1}$ contains no $W_{n}$ and its complement contains no $C_{m}$. To prove $R\left(W_{n}, C_{m}\right) \leq 3 m-2$, let $G$ be graph of order $3 m-2$, and suppose that neither $G$ contains $W_{n}$ nor $\bar{G}$ contains $C_{m}$.

We first show that $G$ contains no $K_{n}$. If $G$ contains a $K_{n}$, then every other vertex in $G$ has at most two neighbors in $K_{n}$; otherwise $G$ contains a $W_{n}$. Since $n-2 \geq m / 2$, by Lemma $10, \bar{G}$ contains a $C_{m}$, a contradiction. Thus, $G$ contains no $K_{n}$.

We next show that $\delta(\bar{G})=m-1$. Let $v \in V(G)$ with $d(v)=\Delta(G)=d$, let $H=G[N(v)]$ and let $Z=V(G) \backslash N[v]$. If $\bar{G}$ is a bipartite graph, say with $V(\bar{G})=(X, Y)$ and $|X| \geq|Y|$, then $|X| \geq 3 m / 2-1 \geq n$, which implies that $G[X]$ contains a $K_{n}$, a contradiction. Thus, $\bar{G}$ is nonbipartite. If $\delta(\bar{G}) \geq m$, then by Lemmas 4 and $6, \bar{G}$ contains $C_{m}$, a contradiction. If $\delta(\bar{G}) \leq m-2$, then $d(v) \geq 2 m-1$, that is, $|H| \geq 2 m-1$. Since $\bar{H}$ has no $C_{m}, H$ contains a $C_{n}$ by Theorem 1 , which together with $v$ forms a $W_{n}$ in $G$, a contradiction. Thus, we have $\delta(\bar{G})=m-1$ and $d=2 m-2$.

We next show that $H$ and $\bar{H}$ are both nonbipartite. First assume that $H$ is a bipartite graph, say with $V(H)=(X, Y)$. Then, since $\bar{G}$ contains no $C_{m}$, we have $|X|=|Y|=$ $m-1$ and $e(X, Y) \geq|X||Y|-1$. Because $\delta(\bar{G})=m-1 \geq 5$, there exist two distinct vertices $x_{1}, x_{2} \in X$ such that $x_{1} z_{1}, x_{2} z_{2} \in E(\bar{G})$, where $z_{1}, z_{2} \in Z$. If $z_{1}=z_{2}$, then $\bar{G}\left[X \cup\left\{z_{1}\right\}\right]$ contains a $C_{m}$; and if $z_{1} \neq z_{2}$, then noting that $z_{1}, z_{2} \in N_{\bar{G}}(v)$, we see that $\bar{G}\left[X \cup\left\{z_{1}, z_{2}, v\right\}\right]$ has a $C_{m}$. Hence, $H$ is nonbipartite.

Now suppose $\bar{H}$ is a bipartite graph. Let $V(\bar{H})=(X, Y)$ and $|X| \geq|Y|$. Since $G$ has no $K_{n}$, we get $|X \cup\{v\}| \leq n-1$, hence $|X| \leq n-2$ and $|Y| \geq 2 m-n \geq m / 2+1$. If $\kappa(H) \geq 2$, then $H$ has two independent edges between $X$ and $Y$. Since both $G[X]$ and $G[Y]$ are complete graphs, $H$ contains a $C_{n}$, a contradiction. Let now $\kappa(H) \leq 1$. Then there exists a vertex $w$ such that $\bar{H}-w$ is a complete bipartite graph in which each partite set has at least $m / 2$ vertices. Since $m$ is even, $\bar{H}$ contains a $C_{m}$, a contradiction. Therefore, $\bar{H}$ is also nonbipartite.

If $|E(\bar{H})| \geq d(d-1) / 4+1 / 2$, then by Lemmas 3 and $8, \bar{H}$ contains a $C_{m}$, a contradiction. Thus, $|E(\bar{H})|<d(d-1) / 4+1 / 2$. Since $m$ is even and $d=2 m-2$, 
we have $d \equiv 2(\bmod 4)$. Thus, $|E(\bar{H})| \leq d(d-1) / 4-1 / 2$, and hence $|E(H)| \geq$ $d(d-1) / 4+1 / 2$. By Lemmas 3 and $8, H$ is weakly pancyclic with $g(H)=3$ and $c(H) \geq m$. Let $C=x_{1} x_{2} \ldots x_{r} x_{1}$ be a longest cycle in $H$ and $V(H) \backslash V(C)=Y=$ $\left\{y_{1}, y_{2}, \ldots, y_{d-r}\right\}$. Then $m \leq r \leq n-1$ and $d-r \geq 2 m-n-1 \geq m / 2$. By Lemma $13,\left|N_{C}\left(y_{i}\right) \cup N_{C}\left(y_{j}\right)\right| \leq\lfloor|C| / 2\rfloor+1$, and so $\left|N_{\bar{G}}\left(y_{i}\right) \cap N_{\bar{G}}\left(y_{j}\right) \cap V(C)\right| \geq$ $\lceil|C| / 2\rceil-1 \geq m / 2-1$ for any two distinct vertices $y_{i}, y_{j} \in Y$. If $\mid N_{\bar{G}}\left(y_{i}\right) \cap$ $N_{\bar{G}}\left(y_{j}\right) \cap V(C) \mid \geq m / 2$ for two distinct vertices $y_{i}, y_{j} \in Y$, say $y_{1}, y_{m / 2}$ are two such vertices. Then we can choose $m / 2$ vertices $x_{i_{1}}, x_{i_{2}}, \ldots, x_{i_{m / 2}}$ from $V(C)$ such that $x_{i_{j}} \in N_{\bar{G}}\left(y_{j}\right) \cap N_{\bar{G}}\left(y_{j+1}\right)$ for $1 \leq j \leq m / 2-1$ and $x_{i_{m / 2}} \in N_{\bar{G}}\left(y_{m / 2}\right) \cap$ $N_{\bar{G}}\left(y_{1}\right)$, implying that $y_{1} x_{i_{1}} y_{2} x_{i_{2}} y_{3} \ldots y_{m / 2} x_{i_{m / 2}} y_{1}$ is a $C_{m}$ in $\bar{G}$, a contradiction. Thus, we have $\left|N_{\bar{G}}\left(y_{i}\right) \cap N_{\bar{G}}\left(y_{j}\right) \cap V(C)\right|=m / 2-1$ for any two distinct vertices $y_{i}, y_{j} \in Y$. By Lemma 13, we have $r=m, d-r=m-2$ and $\bar{G}[Y]=K_{m-2}$. Let $x^{\prime} \in N_{\bar{G}}\left(y_{1}\right) \cap N_{\bar{G}}\left(y_{2}\right) \cap V(C)$ and $x^{\prime \prime} \in N_{\bar{G}}\left(y_{2}\right) \cap N_{\bar{G}}\left(y_{3}\right) \cap V(C)-\left\{x^{\prime}\right\}$. Then $\bar{G}\left[Y \cup\left\{x^{\prime}, x^{\prime \prime}\right\}\right]$ contains a $C_{m}$, our final contradiction. This completes the proof of Theorem 10.

\subsection{Proof of Theorem 11}

Since $W_{2,3}=K_{5}$, using Lemma 1 we see that Theorem 11 holds for $n=3$, and so we may assume that $n \geq 5$. Because neither $4 K_{m-1}$ contains a $C_{m}$ nor its complement contains a $W_{2, n}$, we get that $R\left(C_{m}, W_{2, n}\right) \geq 4 m-3$. So it suffices to show $R\left(C_{m}, W_{2, n}\right) \leq 4 m-3$. Let $G$ be a graph of order $4 m-3$ with $m \geq 9 n / 8+1$. Suppose that $G$ contains no $W_{2, n}$ and that $\bar{G}$ contains no $C_{m}$.

We distinguish the following two cases.

Case 1. $\bar{G}$ contains no $2 K_{\lceil m / 2\rceil}$.

If $\bar{G}$ is bipartite, then $\alpha(\bar{G}) \geq 2 m-1 \geq n+2$, which implies that $G$ contains $W_{2, n}$, a contradiction. So $\bar{G}$ is nonbipartite. If $\delta(\bar{G}) \geq(4 m-1) / 3$, then $\bar{G}$ contains $C_{m}$ by Lemmas 4 and 6 , a contradiction. Hence, $\delta(\bar{G}) \leq(4 m-2) / 3$ and so $\Delta(G) \geq$ $(8 m-10) / 3$. Let $u$ be a vertex with $d_{G}(u)=\Delta(G)=d$ and let $G_{u}=G[N(u)]$.

Since $\left|G_{u}\right| \geq 2 n+3$ and $G$ has no $W_{2, n}, \overline{G_{u}}$ is nonbipartite. We first show that $\delta\left(\overline{G_{u}}\right) \leq(d+1) / 3$. To the contrary, assume $\delta\left(\overline{G_{u}}\right) \geq(d+2) / 3$. Then by Lemma 4 , $\overline{G_{u}}$ is weakly pancyclic with $g\left(\overline{G_{u}}\right) \leq 4$. If $\kappa\left(\overline{G_{u}}\right) \geq 2$, then by Lemma $6, c\left(\overline{G_{u}}\right) \geq$ $2 \delta\left(\overline{G_{u}}\right) \geq m$, so then $\overline{G_{u}}$ contains a $C_{m}$, a contradiction. Assume $\kappa\left(\overline{G_{u}}\right) \leq 1$. Then for some $w \in V\left(G_{u}\right), V\left(G_{u}\right) \backslash\{w\}$ can be partitioned into two parts $V^{\prime}, V^{\prime \prime}$ such that $e_{G}\left(V^{\prime}, V^{\prime \prime}\right)=\left|V^{\prime}\right|\left|V^{\prime \prime}\right|$. Assume that $\left|V^{\prime}\right| \geq\left|V^{\prime \prime}\right|$ and choose $w$ under these restrictions such that $\left|V^{\prime}\right|-\left|V^{\prime \prime}\right|$ is as large as possible. It is obvious that $\left|V^{\prime \prime}\right| \geq$ $\delta\left(\overline{G_{u}}\right) \geq\lceil m / 2\rceil$. Noting that $m \geq n+2 \geq 7$ and $d \geq\lceil(8 m-10) / 3\rceil$, we get that $d \geq 2 m+2$, and hence $\left|V^{\prime}\right| \geq m+1$. If $\delta\left(\bar{G}\left[V^{\prime}\right]\right) \geq\left(\left|V^{\prime}\right|+1\right) / 2$, then by Lemma $2, \bar{G}\left[V^{\prime}\right]$ contains a $C_{m}$, a contradiction. Thus there exists some $u^{\prime} \in V^{\prime}$ such that $d_{V^{\prime}}\left(u^{\prime}\right)=\Delta\left(G\left[V^{\prime}\right]\right)>\left(\left|V^{\prime}\right|-3\right) / 2 \geq n / 2$. If $G\left[V^{\prime \prime}\right]$ has at least one edge, then $G$ has a $C_{n}$ in $N\left(u^{\prime}\right)$ which together with $u, u^{\prime}$ forms a $W_{2, n}$ in $G$. So $\bar{G}\left[V^{\prime \prime}\right]$ is a complete graph. In this case, $\left|V^{\prime \prime}\right| \leq m-1$, since $\bar{G}$ has no $C_{m}$. Thus, $\left|V^{\prime}\right|=d-1-\left|V^{\prime \prime}\right| \geq$ $m+2$ and $d_{V^{\prime}}\left(u^{\prime}\right)>\left(\left|V^{\prime}\right|-3\right) / 2$ and $d_{V^{\prime}}\left(u^{\prime}\right) \geq\lceil m / 2\rceil \geq n / 2+1$. Since $\bar{G}$ has no $2 K_{\lceil m / 2\rceil}, G$ has at least one edge in $N_{V^{\prime}}\left(u^{\prime}\right)$, and so $G$ has also a $C_{n}$ in $N\left(u^{\prime}\right)$ which 
together with $u, u^{\prime}$ forms a $W_{2, n}$ in $G$. Therefore, $\delta\left(\overline{G_{u}}\right) \leq(d+1) / 3$, implying that $\Delta\left(G_{u}\right) \geq(2 d-4) / 3$.

Let $v$ be a vertex of $G_{u}$ with $d_{G_{u}}(v)=\Delta\left(G_{u}\right)=h$, and set $H=G_{u}[N(v)]$. Then $h \geq 16(m-2) / 9$. If $H$ contains a $C_{n}$, then this $C_{n}$ together with $u, v$ forms a $W_{2, n}$ in $G$, a contradiction. Hence, $H$ contains no $C_{n}$.

We are now going to show that $H$ and $\bar{H}$ are both nonbipartite. First assume that $H$ is bipartite, say with $V(H)=(X, Y)$ and $|X| \geq|Y|$. Then $|X| \leq m-1$; otherwise $\bar{H}$ has a $C_{m}$. Thus, $|Y| \geq h-(m-1) \geq(7 m-23) / 9$. If $m \geq 8$, then we have $|Y| \geq\lceil m / 2\rceil$; if $m=7$, then since $d \geq(8 m-10) / 3$ and $h \geq(2 d-4) / 3$, we have $h \geq 10$ and so $|Y| \geq 4=\lceil m / 2\rceil$. Thus, $\bar{H}$ always contains $2 K_{\lceil m / 2\rceil}$, a contradiction. Therefore, $H$ is nonbipartite.

Next suppose that $\bar{H}$ is bipartite, say with $V(\bar{H})=(X, Y)$ and $|X| \geq|Y|$. Since $h \geq 16(m-2) / 9 \geq 16((9 n / 8+1)-2) / 9=2 n-16 / 9$, then $h \geq\lceil 2 n-16 / 9\rceil=2 n-1$. Hence, $|X| \geq n$ and $G[X]$ contains a $C_{n}$, a contradiction. Thus, $\bar{H}$ is also nonbipartite.

Noting that $m \geq 9 n / 8+1, n \geq 5$ and $h \geq 16(m-2) / 9$, we have $\lfloor h / 2\rfloor+1 \geq n$ and $h \geq 7$. If $|E(H)| \geq(h+1)(h-1) / 4-1$, then $|E(H)| \geq(((\lfloor h / 2\rfloor+1)-1)(h-$ $1)+1) / 2$. Thus, by Lemmas 3 and $8, H$ contains a $C_{n}$, a contradiction. This implies that $|E(\bar{H})|>(h-1)^{2} / 4+1$. By Lemma $3, \bar{H}$ is weakly pancyclic with $g(\bar{H})=3$. If $c(\bar{H}) \leq\lfloor h / 2\rfloor$, then $|E(\bar{H})|<(h+1)(h-1) / 4-1$ by Lemma 8 , which implies that $|E(\bar{H})|>(h-1)^{2} / 4+1$, and $c(H) \geq n$ by Lemma 9 . Thus, $H$ contains a $C_{n}$ by Lemma 3, a contradiction. Therefore, $\bar{H}$ is weakly pancyclic with $g(\bar{H})=3$ and $c(\bar{H}) \geq\lfloor h / 2\rfloor+1$.

Let $C=x_{1} x_{2} \ldots x_{r} x_{1}$ be a longest cycle in $\bar{H}$, and let $Y=\left\{y_{1}, y_{2}, \ldots, y_{h-r}\right\}=$ $V(\bar{H}) \backslash V(C)$. Then $\lfloor h / 2\rfloor+1 \leq r \leq m-1$ and $h-r \geq h-m+1$. If $n \geq 7$, then it is easy to check that $|Y| \geq(n+1) / 2$; if $n=5$, we have $m \geq 7$ and $|Y| \geq$ $\lceil(7 m-23) / 9\rceil \geq 3$, and we also get that $|Y| \geq(n+1) / 2$. By Lemma 13 , any two vertices of $Y$ have at least $\lceil r / 2\rceil-1$ common nonadjacent vertices in $C$. It is easy to check that $\lceil r / 2\rceil-1 \geq r / 2-1 \geq(\lfloor h / 2\rfloor+1) / 2-1 \geq h / 4-3 / 4>(n-3) / 2$. Since $n$ is odd, $\lceil r / 2\rceil-1 \geq(n-1) / 2$. By Lemmas 14 and $15, H$ is bipartite, a contradiction. This completes Case 1.

Case 2. $\bar{G}$ contains $2 K_{\lceil m / 2\rceil}$.

We first deal with the subcase that $\kappa(\bar{G}) \leq 2$. We assume that $\{u, w\}$ is a cut set and that $V(\bar{G}) \backslash\{u, w\}=X \cup Y$ with $|X| \geq|Y|$ and $E_{\bar{G}}(X, Y)=\emptyset$. Obviously, $|X| \geq 2 m-2$. If $G[X]$ contains $W_{n}$, then $G[X \cup\{y\}]$ contains $W_{2, n}$ for any $y \in Y$, and hence $|X| \leq 3 m-3$ by Theorem 3. Thus, we have $2 m-2 \leq|X| \leq 3 m-3$ and $|Y| \geq m-2$. If $|X| \geq 2 m-1$, then $G[X]$ contains $C_{n}$ by Theorem 1. If $y^{\prime} y^{\prime \prime} \in E(G[Y])$, then $G\left[X \cup\left\{y^{\prime}, y^{\prime \prime}\right\}\right]$ contains $W_{2, n}$, and so $\bar{G}[Y]$ is a complete graph. Since $\bar{G}$ has no $C_{m},|Y| \leq m-1$. If $|Y|=m-2$, then $\bar{G}[Y]=K_{m-2}$. Since $\bar{G}[Y \cup\{u, w\}]$ has no $C_{m}$, we may assume $u y \in E(G)$ for some $y \in Y$. By Theorem 3 , $G[X \cup\{u\}]$ contains $W_{n}$, which implies that $G[X \cup\{u, y\}]$ has $W_{2, n}$, a contradiction. If $|Y|=m-1$, then $\bar{G}[Y]=K_{m-1}$. Since $\bar{G}[Y \cup\{u, w\}]$ contains no $C_{m}$, we can choose $y \in Y$ such that $u y, w y \in E(G)$. By Theorem 3, $G[X \cup\{u, w\}]$ contains $W_{n}$, which implies that $G[X \cup\{u, w, y\}]$ contains $W_{2, n}$, again a contradiction. Therefore, we conclude that $|X|=2 m-2$ and $|Y|=2 m-3$. By Lemma 16, $G[Y \cup\{u, w\}]$ contains $W_{4}$. Assuming that $w$ is not the hub of the $W_{4}$, then $G[Y \cup\{u\}]$ has a triangle 
$v y^{\prime} y^{\prime \prime}$, where $y^{\prime}, y^{\prime \prime} \in Y$. If $G[X]$ contains $C_{n}$, then $G\left[X \cup\left\{y^{\prime}, y^{\prime \prime}\right\}\right]$ contains $W_{2, n}$, and hence $G[X]$ contains no $C_{n}$. By Theorem $1, G[X \cup\{u\}]$ contains $C_{n}$, which implies that $G[X]$ contains $P_{n-1}$ and any $C_{n}$ in $G[X \cup\{u\}]$ contains $u$. If $v=u$, then any $C_{n}$ in $G[X \cup\{u\}]$ together with $y^{\prime}, y^{\prime \prime}$ forms $W_{2, n}$, and if $v \neq u$, then any $P_{n-1}$ in $G[X]$ together with $v, y^{\prime}, y^{\prime \prime}$ forms $W_{2, n}$ in $G$, a contradiction. Henceforth, we may assume that $\kappa(\bar{G}) \geq 3$.

We set $A, B$ as the vertex sets of the $2 K_{\lceil m / 2\rceil}$. Since $m \geq 9 n / 8+1, m \geq n+2$ and $\lceil m / 2\rceil \geq\lceil(n+2) / 2\rceil=(n+3) / 2 \geq 4$. By Lemma $12, \bar{G}$ contains three disjoint paths joining $A$ and $B$, denoted by $Q_{i}=a_{i} c_{i 1} c_{i 2} \ldots c_{i p_{i}} b_{i}$, where $1 \leq i \leq 3, a_{i} \in A$, $b_{i} \in B$ and $c_{i j} \notin A \cup B$ for $1 \leq j \leq p_{i}$. It is obvious that $p_{i} \geq 0$ and $p_{i}+2$ is the order of $Q_{i}$ for $1 \leq i \leq 3$. We choose three such disjoint paths $Q_{1}, Q_{2}, Q_{3}$ from $\bar{G}$ in such a way that $p_{1}+p_{2}+p_{3}$ is as small as possible. Without loss of generality, we may assume $p_{1} \geq p_{2} \geq p_{3}$.

If $p_{2}+p_{3} \leq m-4$, then it is easy to check that $\bar{G}$ contains a $C_{m}$, a contradiction, implying that $p_{2}+p_{3} \geq m-3 \geq 4$ and $p_{2} \geq 2$. If $p_{1} \geq 7$, then $c_{12} c_{14} \in E(G)$; otherwise $Q_{1}^{\prime}=a_{1} c_{11} c_{12} c_{14} \ldots c_{1 p_{1}} b_{1}$ is a path shorter than $Q_{1}$ in $\bar{G}$ and $Q_{1}^{\prime}, Q_{2}, Q_{3}$ are also three disjoint paths joining $A$ and $B$, contradicting the choice of $Q_{1}, Q_{2}, Q_{3}$. For the same reason, to avoid a path $Q_{1}^{\prime}$ which is shorter than $Q_{1}$ and together with $Q_{2}, Q_{3}$ forms three disjoint paths joining $A$ and $B$ in $\bar{G}, G$ contains a complete multipartite graph with five partite sets: $\left\{c_{12}\right\},\left\{c_{14}\right\},\left\{c_{16}\right\}, A \backslash\left\{a_{2}, a_{3}\right\}, B \backslash\left\{b_{2}, b_{3}\right\}$. Since both $\left|A \backslash\left\{a_{2}, a_{3}\right\}\right|$ and $\left|B \backslash\left\{b_{2}, b_{3}\right\}\right|$ are at least $(n-1) / 2$, then $G$ contains a $W_{2, n}$, a contradiction. This implies that $p_{1} \leq 6$.

By the choice of $Q_{1}, Q_{2}, Q_{3}$, we see that every vertex of $A \backslash\left\{a_{2}, a_{3}\right\}$ is adjacent to every vertex of $B \backslash\left\{b_{2}, b_{3}\right\}$. Furthermore, if $p_{i} \geq 1$, then $a_{i}$ is adjacent to every vertex of $V\left(Q_{i}\right) \backslash\left\{a_{i}, c_{i 1}\right\}, c_{i 1}$ is adjacent to every vertex of $V\left(Q_{i}\right) \backslash\left\{a_{i}, c_{i 1}, c_{i 2}\right\}, c_{i 2}$ is adjacent to every vertex of $V\left(Q_{i}\right) \backslash\left\{c_{i 1}, c_{i 2}, c_{i 3}\right\}, \ldots, b_{i}$ is adjacent to every vertex of $V\left(Q_{i}\right) \backslash\left\{c_{i p_{i}}, b_{i}\right\}$, where $1 \leq i \leq 3$. If $p_{i} \geq 2$, then for $j \geq 2, c_{i j}$ is adjacent to every vertex of $A \backslash\left\{a_{1}, a_{2}, a_{3}\right\}$; for $j \leq p_{i}-1, c_{i j}$ is adjacent to every vertex of $B \backslash\left\{b_{1}, b_{2}, b_{3}\right\}$. For $1 \leq i<s \leq 3$, if $\lfloor m / 2\rfloor \leq j+t \leq m-2$, then $c_{i j}$ is adjacent to $c_{s t}$. This is because, if $c_{i j} c_{s t} \in E(\bar{G})$, then $a_{i} c_{i 1} \ldots c_{i j} c_{s t} \ldots c_{s 1} a_{s}$ is a path which together with $m-2-j-t$ vertices of $A \backslash\left\{a_{i}, a_{s}\right\}$ forms a $C_{m}$ in $\bar{G}$, a contradiction. For $1 \leq i<s \leq 3$, if $\lfloor m / 2\rfloor \leq\left(p_{i}-j+1\right)+\left(p_{s}-t+1\right) \leq m-2$, then $c_{i j}$ is adjacent to $c_{s t}$. This is because, if $c_{i j} c_{s t} \in E(\bar{G})$, then $b_{i} c_{i p_{i}} \ldots c_{i j} c_{s t} \ldots c_{s p_{s}} b_{s}$ is a path which together with $m+j+t-p_{i}-p_{s}-4$ vertices of $B \backslash\left\{b_{i}, b_{s}\right\}$ forms a $C_{m}$ in $\bar{G}$, a contradiction. We can also determine whether $c_{i j}$ is adjacent to $a_{s}$ or $b_{s}$ under similar conditions. In the following, through a tedious but straightforward case distinction, we will always find a $W_{2, n}$ in $G$, which is a contradiction and confirms our claim. Unless specifically mentioned, the existence of the edges of the $W_{2, n}$ that we will find each time is validated by the above arguments.

Set $A \backslash\left\{a_{1}, a_{2}, a_{3}\right\}=\left\{a_{4}, a_{5}, \ldots, a_{\lceil m / 2\rceil}\right\}$ and $B \backslash\left\{b_{1}, b_{2}, b_{3}\right\}=\left\{b_{4}, b_{5}, \ldots, b_{\lceil m / 2\rceil}\right\}$. If $\left(p_{1}, p_{2}\right)=(6,6),(6,5)$, then $7 \leq m \leq p_{2}+p_{3}+3 \leq 2 p_{2}+3 \leq$ 15. We see that $G$ contains a $W_{2, n}=\left\{c_{12}\right\}+\left\{c_{14}\right\}+C_{n}$, where $C_{n}=$ $a_{4} c_{x} b_{4} a_{5} b_{5} \ldots a_{(n+3) / 2} b_{(n+3) / 2} a_{1} b_{1} a_{4}$, where $c_{x}=c_{23}$ for $10 \leq m \leq 11$, and $c_{x}=c_{22}$ for $8 \leq m \leq 9$ or $12 \leq m \leq 15$. For $m=7$, either $a_{1} c_{21} \in E(G)$ or $c_{14} c_{22} \in E(G)$; otherwise $\bar{G}$ contains a $C_{7}=a_{1} c_{11} c_{12} c_{13} c_{14} c_{22} c_{21} a_{1}$, a contradic- 
tion. Thus, $G$ contains a $W_{2,5}=\left\{c_{12}\right\}+\left\{c_{14}\right\}+C_{5}$, where $C_{5}=a_{1} c_{21} b_{4} a_{4} b_{1} a_{1}$ if $a_{1} c_{21} \in E(G)$, and $C_{5}=a_{4} c_{22} b_{4} a_{1} b_{1} a_{4}$ if $c_{14} c_{22} \in E(G)$. If $\left(p_{1}, p_{2}\right)=$ $(6,4),(6,3),(5,5),(5,4),(5,3)$, then $7 \leq m \leq 2 p_{2}+3 \leq 13$. We see that $G$ contains a $W_{2, n}=\left\{c_{12}\right\}+\left\{c_{14}\right\}+C_{n}$, where $C_{n}=a_{4} c_{22} b_{4} a_{5} b_{5} \ldots a_{(n+3) / 2} b_{(n+3) / 2} a_{1} b_{1} a_{4}$ for $8 \leq m \leq 13$. For $m=7$, we can obtain the same $W_{2,5}$ as in the previous case. If $\left(p_{1}, p_{2}\right)=(6,2),(5,2)$, then $m=7$ and $p_{3}=2$. In this case, $G$ contains a $W_{2,5}=\left\{c_{12}\right\}+\left\{c_{14}\right\}+C_{5}$, where $C_{5}=a_{4} c_{22} c_{32} c_{21} b_{4} a_{4}$. For the remainder we may assume that $p_{1} \leq 4$.

If $\left(p_{1}, p_{2}, p_{3}\right)=(4,4,4)$ and $m \neq 8$, or if $\left(p_{1}, p_{2}, p_{3}\right)=(4,4,3)$, then $m \leq 11$ and $G$ contains a $W_{2, n}=\left\{c_{12}\right\}+\left\{c_{22}\right\}+C_{n}$, where $C_{n}=$ $a_{4} c_{32} c_{14} c_{31} b_{4} a_{5} b_{5} \ldots a_{(n+3) / 2} b_{(n+3) / 2} a_{4}$. If $\left(p_{1}, p_{2}, p_{3}\right)=(4,4,4)$ and $m=8$, then $n=5$ and $G$ contains a $W_{2,5}=\left\{a_{4}\right\}+\left\{b_{4}\right\}+C_{5}$, where $C_{5}=c_{12} c_{22} c_{32} c_{23} c_{33} c_{12}$. If $\left(p_{1}, p_{2}, p_{3}\right)=(4,4,2)$, then $m \leq 9$. We see that $G$ contains a $W_{2, n}=$ $\left\{c_{12}\right\}+\left\{c_{22}\right\}+C_{n}$, where $C_{n}=a_{4} c_{32} c_{14} c_{31} b_{4} a_{5} b_{5} \ldots a_{(n+3) / 2} b_{(n+3) / 2} a_{4}$ for the cases $m=8,9$ or the case that $m=7$ and $c_{14} c_{32} \in E(G)$. If $m=7$ and $c_{24} c_{32} \in E(G)$, since $c_{14} c_{32}$ and $c_{24} c_{32}$ are symmetrical, we can also obtain a $W_{2, n}$ in $G$. Thus, $c_{14} c_{32}, c_{24} c_{32} \in E(\bar{G})$, and then $\bar{G}$ contains a $C_{7}=b_{1} c_{14} c_{32} c_{24} b_{2} b_{3} b_{4} b_{1}$, a contradiction. If $\left(p_{1}, p_{2}, p_{3}\right)=(4,4,1)$, then $m \leq 8$ and $n=5$. For $m=7, G$ contains a $W_{2,5}=\left\{c_{21}\right\}+\left\{c_{31}\right\}+C_{5}$, where $C_{5}=c_{14} c_{23} c_{13} c_{24} c_{12} c_{14}$. For $m=8, G$ contains a $W_{2,5}=\left\{a_{3}\right\}+\left\{b_{3}\right\}+C_{5}$, where $C_{5}=c_{13} c_{22} c_{12} c_{23} c_{11} c_{13}$. If $\left(p_{1}, p_{2}, p_{3}\right)=$ $(4,4,0)$, then $m=7$ and $G$ contains a $W_{2,5}=\left\{c_{21}\right\}+\left\{b_{3}\right\}+C_{5}$, where $C_{5}=$ $c_{14} c_{23} c_{13} c_{24} c_{12} c_{14}$. If $\left(p_{1}, p_{2}, p_{3}\right)=(4,3,3),(3,3,3)$, then $m \leq 9$ and $G$ contains a $W_{2, n}=\left\{c_{12}\right\}+\left\{c_{22}\right\}+C_{n}$, where $C_{n}=a_{4} c_{33} c_{31} b_{4} a_{5} b_{5} \ldots a_{(n+3) / 2} b_{(n+3) / 2} c_{32} a_{4}$. If $\left(p_{1}, p_{2}, p_{3}\right)=(4,3,2)$, then $m \leq 8$ and $n=5$. For the case $m=8$ or the case $m=7$ and $c_{11} c_{31} \in E(G), G$ contains a $W_{2,5}=\left\{c_{13}\right\}+\left\{c_{22}\right\}+C_{5}$, where $C_{5}=c_{31} c_{11} c_{32} a_{4} b_{4} c_{31}$. For $m=7$ and $c_{11} c_{31} \in E(\bar{G})$, we have $b_{3} c_{14} \in E(G)$; otherwise $c_{11} c_{12} c_{13} c_{14} b_{3} c_{32} c_{31} c_{11}$ is a $C_{7}$ in $\bar{G}$, a contradiction. Then $G$ contains a $W_{2,5}=\left\{c_{12}\right\}+\left\{c_{22}\right\}+C_{5}$, where $C_{5}=b_{4} c_{31} c_{14} b_{3} a_{4} b_{4}$. If $\left(p_{1}, p_{2}, p_{3}\right)=(4,3,1)$, then $m=7$. If $a_{3} c_{11}, b_{3} c_{14} \in E(\bar{G})$, then $c_{11} c_{12} c_{13} c_{14} b_{3} c_{31} a_{3} c_{11}$ is a $C_{7}$ in $\bar{G}$, a contradiction. By symmetry, we may assume that $a_{3} c_{11} \in E(G)$, and $G$ contains a $W_{2,5}=\left\{c_{13}\right\}+\left\{c_{22}\right\}+C_{5}$, where $C_{5}=a_{3} c_{11} b_{3} a_{4} b_{4} a_{3}$. If $\left(p_{1}, p_{2}, p_{3}\right)=(4,2,2)$, then $m=7$. If $a_{3} c_{12}, a_{4} c_{21} \in E(\bar{G})$, then $a_{1} c_{11} c_{12} a_{3} a_{4} c_{21} a_{2} a_{1}$ is a $C_{7}$ in $\bar{G}$, a contradiction. Hence, either $a_{3} a_{12} \in E(G)$ or $a_{4} a_{21} \in E(G)$. Thus, $G$ contains a $W_{2,5}=\left\{c_{12}\right\}+\left\{c_{21}\right\}+C_{5}$, where $C_{5}=a_{x} c_{32} b_{2} c_{31} b_{3} a_{x}, a_{x}=a_{3}$ if $a_{3} a_{12} \in E(G)$, and $a_{x}=a_{4}$ if $a_{4} a_{21} \in E(G)$. If $\left(p_{1}, p_{2}, p_{3}\right)=(3,3,2)$, then $m \leq 8$ and $n=5$. For the case $m=8$ or the case $m=7$ and $a_{3} c_{21} \in E(G), G$ contains a $W_{2,5}=\left\{c_{12}\right\}+\left\{c_{21}\right\}+C_{5}$, where $C_{5}=a_{3} c_{32} c_{23} c_{31} b_{3} a_{3}$. For $m=7$ and $a_{3} c_{21} \in E(\bar{G})$, we have $b_{3} c_{23} \in E(G)$; otherwise $a_{3} c_{31} c_{32} b_{3} c_{23} c_{22} c_{21} a_{3}$ is a $C_{7}$ in $\bar{G}$, a contradiction. Since $a_{3} c_{21}$ and $b_{3} c_{23}$ are symmetrical, we can also obtain a $W_{2,5}$ if $b_{3} c_{23} \in E(G)$. If $\left(p_{1}, p_{2}, p_{3}\right)=(3,2,2)$, then $m=7$ and $G$ contains a $W_{2,5}=\left\{c_{12}\right\}+\left\{c_{21}\right\}+C_{5}$, where $C_{5}=a_{3} c_{32} b_{2} c_{31} b_{3} a_{3}$. If $\left(p_{1}, p_{2}, p_{3}\right)=(2,2,2)$, then $m=7$ and $G$ contains a $W_{2,5}=\left\{c_{11}\right\}+\left\{c_{21}\right\}+C_{5}$, where $C_{5}=a_{3} c_{32} b_{2} c_{31} b_{3} a_{3}$. Since $p_{2}+p_{3} \geq 4$, we have considered all the possible combinations of values for $\left(p_{1}, p_{2}, p_{3}\right)$, and each time we derived a contradiction. This completes the proof of Case 2 and of Theorem 11. 
Acknowledgments We thank the anonymous referees for useful comments that improved the presentation. This research was supported by NSFC under grant numbers 11071115,11371193 and 11101207 , and by China Scholarship Council (File No. 201306190026).

Open Access This article is distributed under the terms of the Creative Commons Attribution License which permits any use, distribution, and reproduction in any medium, provided the original author(s) and the source are credited.

\section{References}

1. Bollobás, B., Jayawardene, C.J., Yang, J.S., Huang, Y.R., Rousseau, C.C., Zhang, K.M.: On a conjecture involving cycle-complete graph Ramsey numbers. Aust. J. Comb. 22, 63-71 (2000)

2. Bondy, J.A.: Pancyclic graphs. J. Comb. Theory Ser. B 11, 80-84 (1971)

3. Bondy, J.A., Murty, U.S.R.: Graph Theory. Springer, Berlin (2008)

4. Brandt, S.: A sufficient condition for all short cycles. Discret. Appl. Math. 79, 63-66 (1997)

5. Brandt, S., Faudree, R., Goddard, W.: Weakly pancyclic graphs. J. Graph Theory 27, 141-176 (1998)

6. Burr, S.A., Erdős, P.: Generalizations of a Ramsey-theoretic result of Chvátal. J. Graph Theory 7, 39-51 (1983)

7. Chen, Y.J., Cheng, T.C.E., Miao, Z.K., Ng, C.T.: The Ramsey numbers for cycles versus wheels of odd order. Appl. Math. Lett. 22, 1875-1876 (2009)

8. Chen, Y.J., Cheng, T.C.E., Ng, C.T., Zhang, Y.Q.: A theorem on cycle-wheel Ramsey number. Discret. Math. 312, 1059-1061 (2012)

9. Dirac, G.A.: Some theorems on abstract graphs. Proc. Lond. Math. Soc. 2, 69-81 (1952)

10. Dybizbański, J., Dzido, T.: On some Ramsey numbers for quadrilaterals versus wheels. Graphs Comb. 30, 573-579 (2014)

11. Erdős, P., Gallai, T.: On maximal paths and circuits of graphs. Acta Math. Acad. Sci. Hung. 10, 337-356 (1959)

12. Faudree, R.J., Lesniak, L., Schiermeyer, I.: On the circumference of a graph and its complement. Discret. Math. 309, 5891-5893 (2009)

13. Faudree, R.J., Schelp, R.H.: All Ramsey numbers for cycles in graphs. Discret. Math. 8, 313-329 (1974)

14. Jackson, B.: Cycles in bipartite graphs. J. Comb. Theory Ser. B 30, 332-342 (1981)

15. Károlyi, G., Rosta, V.: Generalized and geometric Ramsey numbers for cycles. Theor. Comput. Sci. 263, 87-98 (2001)

16. Radziszowski, S.P.: Small Ramsey numbers. Electron. J. Comb. DS1.14 (2014)

17. Rosta, V.: On a Ramsey type problem of J.A. Bondy and P. Erdős, I and II. J. Comb. Theory Ser. B 15, 94-120 (1973)

18. Shi, L.S.: Ramsey numbers of long cycles versus books or wheels. Euro. J. Comb. 31, 828-838 (2010)

19. Sun, S.Y., Chen, Y.J.: On wheels versus a pentagon Ramsey numbers, submitted

20. Surahmat, Baskoro, E.T., Broersma, H.J.: The Ramsey numbers of large cycles versus small wheels integers. Electron. J. Comb. Number Theory 4(10), 9 (2014)

21. Surahmat, Baskoro, E.T., Tomescu, I.: The Ramsey numbers of large cycles versus wheels. Discret. Math. 306, 3334-3337 (2006)

22. Surahmat, Baskoro, E.T., Tomescu, I.: The Ramsey numbers of large cycles versus odd wheels. Graphs Comb. 24, 53-58 (2008)

23. Surahmat, Baskoro, E.T., Uttunggadewa, S., Broersma, H.J.: An upper bound for the Ramsey number of a cycle of length four versus wheels, LNCS 3330. Springer, Berlin (2005)

24. Surahmat, Tomescu, I., Baskoro, E.T., Broersma, H.J.: On Ramsey numbers of cycles with respect to generalized even wheels, manuscript (2006)

25. Tse, K.K.: On the Ramsey number of the quadrilateral versus the book and the wheel. Aust. J. Comb. 27, 163-167 (2003)

26. Wu, Y.L., Sun, Y.Q., Radziszowski, S.: Wheel and star-critical Ramsey numbers for quadrilateral. http://www.cs.rit.edu/ spr/PUBL/wsr13

27. Wu, Y.L., Sun, Y.Q., Zhang, R., Radziszowski, S.: Ramsey numbers of $C_{4}$ versus wheels and stars. http://www.cs.rit.edu/ spr/PUBL/sur14 
28. Zhang, L.M., Chen, Y.J., Edwin Cheng, T.C.: The Ramsey numbers for cycles versus wheels of even order. Euro. J. Comb. 31, 254-259 (2010)

29. Zhang, Y.B., Broersma, H.J., Chen, Y.J.: A remark on star- $C_{4}$ and wheel- $C_{4}$ Ramsey numbers. Electron. J. Graph Theory Appl. 2, 110-114 (2014)

30. Zhang, Y.B., Zhang, Y.Q., Chen, Y.J.: The Ramsey numbers of wheels versus odd cycles. Discret. Math. 323, 76-80 (2014)

31. Zhou, H.L.: The Ramsey number of an odd cycle with respect to a wheel (in Chinese). J. Math. Shuxue Zazhi (Wuhan), 15, 119-120 (1995) 